\title{
Non-destructive 3D orientational mapping of bone using diffractive X-ray tomography
}

\author{
F. K. Mürer ${ }^{1}$, S. Sanchez ${ }^{2}$, K. Olstad ${ }^{3}$, M. Di Michiel ${ }^{4}$, B. Chattopadhyay ${ }^{1}$, D. W. Breiby ${ }^{1,5}$
}

${ }^{1}$ PoreLab, Department of Physics, Norwegian University of Science and Technology (NTNU), Høgskoleringen 5, 7491 Trondheim, Norway. ${ }^{2}$ Uppsala University, Department of Organismal Biology, Evolutionary Biology Centre, Norbyvägen 18 A, 75236, Uppsala, Sweden. ${ }^{3}$ Faculty of Veterinary Medicine, Department of Companion Animal Clinical Sciences, Norwegian University of Life Sciences, Oslo, Norway. ${ }^{4}$ ESRF-The European Synchrotron, 71 Avenue des Martyrs, 38000 Grenoble, France. ${ }^{5}$ Department of Microsystems, University of South-Eastern Norway (USN), Campus Vestfold, 3184 Borre, Norway.

fredrik.k.murer@ntnu.no

Bone is a strong yet light-weight material, where several mechanical properties originate from the orientation of their molecular components - collagen fibrils mineralized with calcium phosphate in a hydroxyapatite (HA)-like structure. Knowledge of the threedimensional (3D) microscopic orientation arrangements of the mineralized collagen in macroscopic samples, allows for a deeper understanding of the mechanical properties of bone, leading to an improved understanding of bone and cartilage-related diseases such as osteochondrosis and osteoarthritis. The distinct patterns in the HA mineral orientation can also be used to locate embedded fibres of muscle attachments in vertebrates in both modern and fossil bones. This is crucial for reconstructing evolutionary scenarios and biomechanical models of extinct species, for which soft tissues are lost during fossilization.

X-ray diffraction computed tomography (XRD-CT) is an emerging imaging technique, allowing non-destructive 3D mapping of samples with material-specific contrast [1] and has recently also been demonstrated with orientational contrast [2-4]. In this presentation we demonstrate the application of XRD-CT to study the microstructure of different types of bones without destructive sample sectioning. The HA orientation in a tibial cross-section from a fossil stem amniote Discosauriscus austriacus is used to reveal the location of muscle attachments, shown in Figs. 1a and 1b. XRD-CT can also be used to study the HA orientation close to the bonecartilage interface in the developing bone, as illustrated in Figs. 1c and 1d. XRD-CT is becoming a powerful tool that allows studying the orientation of mineralized structures in bone, and is likely to be increasingly used due to the advent of new synchrotron sources and improved numerical methods for tomographic reconstruction.
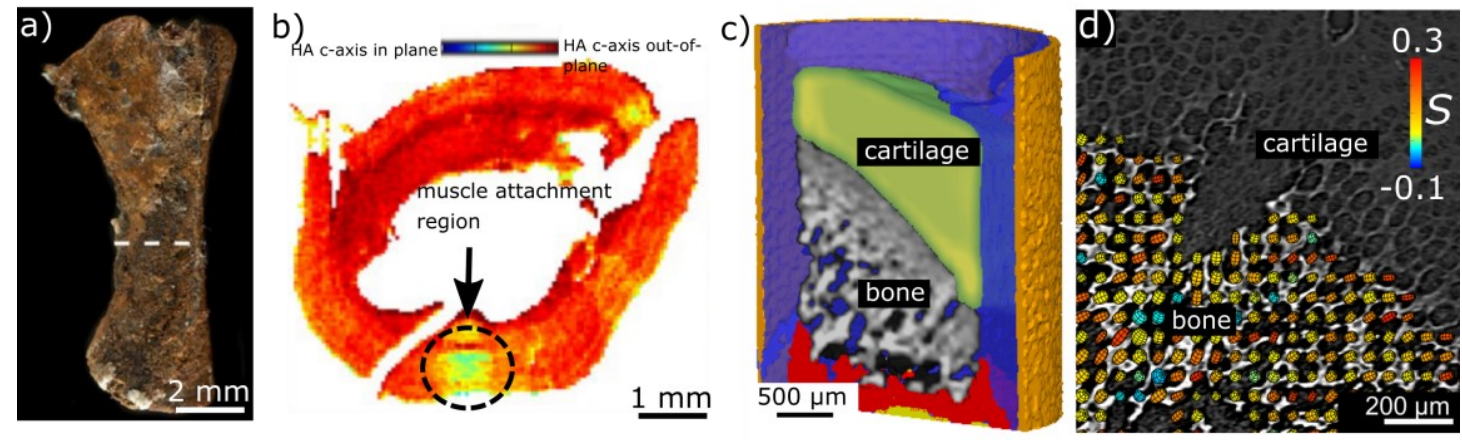

Figure 1. a) Photograph of a tibia from a fossil stem amniote Discosauriscus austriacus. b) Orientational XRD-CT cross-section from the region indicated with a dashed line in a). The colour coding corresponds to the alignment of the HA $c$-axis with the bone long-axis, which is used to identify muscle attachments. c) Material-specific XRD-CT 3D map of a bone-cartilage sample from a pig, placed inside a sample container for the experiment. Colours: yellow - rubber plug, red - cyanoacrylate ("super glue"), blue - ethanol/water solution and orange - polyimide sample container. d) XRD-CT cross-section showing the preferred orientation of the HA crystallite $c$-axis, represented with oriented ellipsoids. The colour coding corresponds to the Hermans' orientation parameter $S$.

[1] Harding, G., Kosanetzky, J. \& Neitzel, U. (1987). Med. Phys. 14, 515.

[2] Liebi, M., Georgiadis, M., Menzel, A., Schneider, P., Kohlbrecher, J., Bunk, O. \& Guizar-Sicairos, M. (2015). Nature. $527,349$.

[3] Mürer, F. K., Sanchez, S., Álvarez-Murga, M., Di Michiel, M., Pfeiffer, F., Bech, M. \& Breiby, D. W. (2018). Sci. Rep. 8, 1.

[4] Mürer, F. K., Chattopadhyay, B., Madathiparambil, A. S., Tekseth, K. R., Di Michiel, M., Liebi, M., Lilledahl, M. B., Olstad, K. \& Breiby, D. W. (2021). Sci. Rep. 11, 1.

Keywords: Biomineralization; X-ray diffraction computed tomography; Orientation mapping.

We thank the Research Council of Norway for financial funding through FRINATEK (\#275182), NANO2021 (\#272248) and its Centres of Excellence funding scheme (\#262644). ESRF-The European Synchrotron is thanked for beamtime at ID15A.

Acta Cryst. (2021), A77, C412 